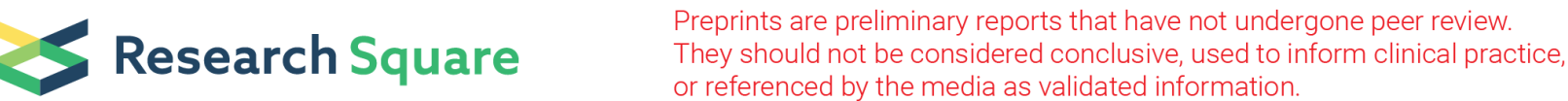

\section{Low-Flow, Low-Gradient Severe Aortic Stenosis: Cardiac Computed Tomography Findings and Clinical Outcomes After Aortic Valve Replacement}

\section{Se Jin Choi}

University of Ulsan College of Medicine

\section{Yura Ahn}

University of Ulsan College of Medicine

Hyun Jung Koo ( $\nabla$ radkoo@amc.seoul.kr)

University of Ulsan College of Medicine

Dae-Hee Kim

University of Ulsan College of Medicine

Soyeon Lim

Ulsan University Hospital

Jun Bum Kim

University of Ulsan College of Medicine

Jong-Min Song

University of Ulsan College of Medicine

Duk-Hyun Kang

University of Ulsan College of Medicine

Jae-Kwan Song

University of Ulsan College of Medicine

Hwa Jung Kim

University of Ulsan College of Medicine

Joon-Won Kang

University of Ulsan College of Medicine

Dong Hyun Yang

University of Ulsan College of Medicine

\section{Research Article}

Keywords: cardiac computed tomographic, Aortic valve calcium, echocardiography, surgical AVR

Posted Date: December 17th, 2021

DOI: https://doi.org/10.21203/rs.3.rs-1151439/v1 
License: (c) (i) This work is licensed under a Creative Commons Attribution 4.0 International License. Read Full License 


\section{Abstract}

Aortic valve calcium scoring by cardiac computed tomographic (CT) has been recommended as an alternative to classify the AS severity, but it is unclear that whether CT findings can predict and have prognostic implication in low-flow, low-gradient aortic stenosis (LF-LG AS), which has fewer benefit from surgery among the AS subtypes. In this study, we examined the clinical and cardiac CT findings of LF-LG AS patients and evaluated factors affecting outcomes after surgical aortic valve replacement (AVR). This study included 511 (66.9 \pm 8.8 years, $55 \%$ men) consecutive patients with severe AS who underwent surgical AVR. Aortic valve area (AVA) was obtained by echocardiography $\left(A V A_{\text {echo }}\right)$ and by CT ( $\left.A V A_{C T}\right)$ using each modalities measurement of the left ventricular outflow tract. Patients with AS were classified as 1$)$ high-gradient severe $(n=438), 2)$ classic LF-LG $(n=18)$, and 3) paradoxical LF-LG $(n=55)$ based on echocardiography. Classic LF-LG AS patients had higher end-systolic and end-diastolic volume indices, lower left ventricular ejection fraction, larger $\mathrm{AVA}_{\text {echo }}$ and $\mathrm{AVA} \mathrm{AT}_{\mathrm{CT}}$, and larger aortic annulus compared to high-gradient severe AS ( $P<0.05$, for all). In classic LF-LG AS group, $27.8 \%$ of patients presented $A V A_{C T} \geq 1.2 \mathrm{~cm}^{2}$. After multivariable adjustment, old age (hazard ratio [HR], 1.04, $P=0.049$ ), high B-type natriuretic peptide (BNP) (HR, 1.005; $P<0.001)$, preoperative atrial fibrillation ( $H R, 2.75 ; P=0.003)$, classic LF-LG AS (HR, 5.53, $P=0.004)$, and small aortic annulus (HR, 0.57; $P=0.002)$ were independently associated with major adverse cardiac and cerebrovascular events (MACCE). The classic LF-LG AS group presented larger $\mathrm{AVA}_{\mathrm{CT}}$ and aortic annulus than those in high-gradient severe AS group and one third of them had $A V A_{C T} \geq 1.2 \mathrm{~cm}^{2}$. Old age, high BNP, atrial fibrillation, classic LF-LG AS, and small aortic annulus were associated with MACCE in severe AS patients after surgical AVR.

\section{Introduction}

Classic low-flow, low-gradient (LF-LG) severe aortic stenosis (AS) is defined by a small aortic valve (AV) area (AVA) on echocardiography ( $A V A_{\text {echo }}<1 \mathrm{~cm}^{2}$ ), a low mean pressure gradient ( $P G<40 \mathrm{mmHg}$ ), and low flow (stroke volume $[\mathrm{SV}]<35 \mathrm{~mL} / \mathrm{m}^{2}$ ). The condition is characterised by low cardiac output due to a reduced left ventricular ejection fraction (LVEF $<50 \%) .{ }^{1-5}$ Conversely, LF-LG AS may occur despite preserved LVEF and is classified as paradoxical LF-LG AS. Assessment of disease severity, management, and prediction of post-surgical outcome in patients with LF-LG AS is currently challenging. Since LF-LG AS presents fewer potential benefits from AV replacement (AVR) and considerable operation risks compared to true-severe AS, a classification for AS is important. ${ }^{5}$

Although the planimetry of the AVA using three-dimensional transoesophageal echocardiography has been reported to be more accurate than transthoracic echocardiography, ${ }^{6}$ measurement issues still remain unresolved. Even in patients with normal systolic LV function, the grading of AS on echocardiography is inconsistent, and this is partly due to reduced SV. ${ }^{7,8}$ In patients with low-flow state, AS severity may be underestimated due to lower mean PG, while incomplete opening of the AV may overestimate stenosis severity because of the reduced opening forces to the $A V .{ }^{9}$ In patients with low-flow state, there can be a discrepancy between the EOA and the PG. Moreover, the continuity equation 
assumes circular LVOT which is elliptical shape, and echocardiography may underestimate LVOT. Additional diagnostic tests, dobutamine stress echocardiography (DSE) ${ }^{2,10}$ and AV calcium score (AVC) obtained by computed tomography (CT) scan ${ }^{11} 12$, have been used for the confirmation of severity and therapeutic guidance, and there is a chance that the patients with severe AS may be reclassified into the moderate range. However, reference standards used in these studies consisted of subjective assessment of the valve severity by cardiac surgeons and the AVC on CT images, which do not reflect hemodynamic severity.

Cardiac CT is recommended as an alternative to assess AS severity when DSE is inconclusive. ${ }^{13}$ However, discrepancies have been reported between the measured AVA on cardiac CT ( $A V A_{C T}$ ) and AVA echo. $^{14,15}$ $A V A_{C T}$ was significantly greater than the $A V A_{\text {echo }}$ calculated by continuity equation, and suggested cut-off of $A V A_{C T}$ for severe AS was $<1.2 \mathrm{~cm}^{2}$. Moreover, CT findings of LF-LG AS and imaging prognostic values remain undefined. Thus, we sought to (i) determine the CT characteristics of LF-LG AS, and (ii) evaluate prognostic factors affecting major adverse cardiovascular and cerebrovascular events (MACCE) of AS after AVR.

\section{Results}

\section{Patient characteristics}

High-gradient severe AS (85.7\% [438/511]) was most common among patients, followed by paradoxical LF-LG AS (10.8\% [55/511]) and classic LF-LG AS (3.5\% [18/511]). (Supplementary Table 1). Half of the patients had tricuspid valves (48.1\% [246/511]) and bicuspid valves were detected in the remaining patients. The median follow-up period for all patients was 4.12 (IQR, 3.19-5.50) years. MACCE occurred in $43(8.4 \%)$ patients, and the overall mortality was $13.9 \%(n=71)$.

Among the groups with high-gradient severe AS, classic LF-LG AS, and paradoxical LF-LG AS, the age of patients were not statistically different $(P=0.93)$ (Table 1$)$. The number of concurrent percutaneous coronary artery intervention or CABG with AVR was highest in classic LF-LG AS group (50\%, $P=0.02)$. BNP was highest in classic LF-LG AS (median $944.5 \mathrm{pg} / \mathrm{mL}, \mathrm{P}<0.001$ ). MACCE was more common in the classic LF-LG AS than in the high-gradient severe AS ( 27.8 vs. $7.8 \%, P=0.01)$. 
Table 1

Clinical and imaging characteristics of high-gradient severe, classic LF-LG, paradoxical LF-LG AS groups $(n=511)$

\begin{tabular}{|c|c|c|c|c|}
\hline Characteristic & $\begin{array}{l}\text { High-gradient } \\
\text { severe AS }\end{array}$ & Classic LF-LG AS & $\begin{array}{l}\text { Paradoxical LF-LG } \\
\text { AS }\end{array}$ & $\begin{array}{l}\mathrm{P}- \\
\text { value }\end{array}$ \\
\hline No. of patients (\%) & 438 (85.7) & $18(3.5)$ & $55(10.8)$ & \\
\hline Age, years & $66.8 \pm 8.8$ & $66.8 \pm 6.2$ & $67.3 \pm 9.4$ & 0.93 \\
\hline Male & $236(53.9)$ & $13(72.2)$ & $31(56.4)$ & 0.30 \\
\hline Body surface area, $\mathrm{m}^{2}$ & $1.6 \pm 0.2$ & $1.7 \pm 0.2$ & $1.6 \pm 0.2$ & 0.56 \\
\hline Hypertension & $231(52.7)$ & $12(66.7)$ & $30(54.5)$ & 0.50 \\
\hline Atrial fibrillation & $65(14.8)$ & $4(22.2)$ & $4(7.3)$ & 0.20 \\
\hline $\mathrm{PCl}$ or $\mathrm{CABG}$ & $94(21.5)$ & $9(50.0)^{*}$ & $14(25.5)$ & 0.02 \\
\hline $\mathrm{BNP}, \mathrm{pg} / \mathrm{mL}$ & $\begin{array}{l}99.5(43.0- \\
280.5)\end{array}$ & $\begin{array}{l}944.5(304.8- \\
3066.0)^{\star}\end{array}$ & $\begin{array}{l}70.0(35.0- \\
190.0)\end{array}$ & $<0.001$ \\
\hline InBNP & $4.6(3.8-5.6)$ & $6.8(5.7-8.0)^{\star}$ & $4.2(3.6-5.2)$ & $<0.001$ \\
\hline \multicolumn{5}{|l|}{ Echocardiography } \\
\hline LVEF, \% & $60.3 \pm 10.0$ & $36.0 \pm 10.3^{*}$ & $62.6 \pm 5.3$ & $<0.001$ \\
\hline Peak velocity, $\mathrm{m} / \mathrm{s}$ & $5.2 \pm 0.7$ & $3.6 \pm 0.5^{\star}$ & $3.5 \pm 0.5^{\dagger}$ & $<0.001$ \\
\hline Peak PG, mmHg & $108.5 \pm 30.5$ & $52.0 \pm 13.1^{*}$ & $50.1 \pm 16.0^{\dagger}$ & $<0.001$ \\
\hline Mean PG, mmHg & $66.5 \pm 19.4$ & $29.8 \pm 7.9^{*}$ & $28.0 \pm 9.5^{\dagger}$ & $<0.001$ \\
\hline LVMI, gm/ m² & $135.4 \pm 35.8$ & $149.6 \pm 31.1$ & $124.9 \pm 36.2$ & 0.03 \\
\hline AV VTI, cm & $124.6 \pm 26.1$ & $94.9 \pm 33.3^{*}$ & $112.0 \pm 27.7^{\dagger}$ & $<0.001$ \\
\hline LVOT VTI, cm & $21.4 \pm 4.1$ & $16.0 \pm 4.4^{*}$ & $21.2 \pm 3.7$ & $<0.001$ \\
\hline LVOT diameter, mm & $21.0 \pm 1.5$ & $21.8 \pm 1.9$ & $21.2 \pm 1.6$ & 0.06 \\
\hline
\end{tabular}

Values are means \pm standard deviations or numbers and percentages in parentheses. ${ }^{\text {SSignificant }}$ difference between patients with severe aortic stenosis and patients with classic low-flow, lowgradient, severe aortic stenosis (classic LF-LG AS) groups. ${ }^{\dagger}$ Significant difference between severe aortic stenosis and paradoxical LF-LG AS groups. AS, aortic stenosis; AVA, aortic valve area; AVC, aortic valve calcium score; BNP, B-type natriuretic peptide; EDVI, end-diastolic volume index; ESVI, endsystolic volume index; LF-LG, low-flow and low-gradient; InBNP, log-transformed B-type natriuretic peptide; LVEF, left ventricular ejection fraction; LVMI, left ventricular mass index; LVOT, left ventricular outflow tract; MACCE, major adverse cardiac and cerebrovascular event; N/A, not available; PG, pressure gradient; SAC, systemic arterial compliance; VTI, velocity time integral; Zva, valvulo-arterial impedance. 


\begin{tabular}{|c|c|c|c|c|}
\hline Characteristic & $\begin{array}{l}\text { High-gradient } \\
\text { severe AS }\end{array}$ & Classic LF-LG AS & $\begin{array}{l}\text { Paradoxical LF-LG } \\
\text { AS }\end{array}$ & $\begin{array}{l}\mathrm{P} \text { - } \\
\text { value }\end{array}$ \\
\hline LVOT diameter/BSA & $12.9 \pm 1.3$ & $13.1 \pm 1.6$ & $13.0 \pm 1.3$ & 0.55 \\
\hline $\mathrm{AVA}_{\mathrm{echo}}, \mathrm{mm}^{2}$ & $61.3 \pm 14.7$ & $66.9 \pm 15.1$ & $69.5 \pm 13.8^{\dagger}$ & $<0.001$ \\
\hline $\mathrm{ESVI}, \mathrm{mL} / \mathrm{m}^{2}$ & $27.7 \pm 16.8$ & $63.4 \pm 27.0^{*}$ & $24.5 \pm 11.4$ & $<0.001$ \\
\hline $\mathrm{EDVI}, \mathrm{mL} / \mathrm{m}^{2}$ & $66.8 \pm 23.7$ & $96.3 \pm 29.3^{*}$ & $64.3 \pm 24.5$ & $<0.001$ \\
\hline $\mathrm{SAC}, \mathrm{mL} / \mathrm{m}^{2} / \mathrm{mmHg}$ & $0.8 \pm 0.3$ & $0.7 \pm 0.3$ & $0.8 \pm 0.3$ & 0.28 \\
\hline $\mathrm{Zva}, \mathrm{mmHg} / \mathrm{mL} / \mathrm{m}^{2}$ & $5.3 \pm 1.6$ & $5.0 \pm 1.9$ & $4.4 \pm 1.4^{+}$ & $<0.001$ \\
\hline \multicolumn{5}{|l|}{ CT findings } \\
\hline Valve morphology & & & & 0.04 \\
\hline Tricuspid & $204(46.6)$ & $14(77.8)$ & $28(50.9)$ & \\
\hline Bicuspid with raphe & $106(24.2)$ & $3(16.7)$ & $17(30.9)$ & \\
\hline Bicuspid without raphe & $128(29.2)$ & $1(5.6)$ & $10(18.2)$ & \\
\hline AVC & $3027.2 \pm 1872.0$ & $2895.5 \pm 1624.5$ & $2363.1 \pm 1605.8^{*}$ & 0.04 \\
\hline LVOT mean diameter & $24.8 \pm 2.9$ & $27.1 \pm 2.7^{\star}$ & $24.7 \pm 2.6$ & 0.003 \\
\hline $\mathrm{AVA}_{\mathrm{CT}}, \mathrm{mm}^{2}$ & $84.9 \pm 23.4$ & $100.8 \pm 22.7^{\star}$ & $94.2 \pm 25.0^{\dagger}$ & 0.001 \\
\hline $\mathrm{AVA}_{\text {plani, }}, \mathrm{mm}^{2}$ & $87.2 \pm 23.2$ & $99.7 \pm 25.5^{\star}$ & $97.5 \pm 27.1^{\dagger}$ & 0.003 \\
\hline \multicolumn{5}{|l|}{ Aortic annulus } \\
\hline Circularity, \% & $81.6 \pm 7.6$ & $77.4 \pm 6.6$ & $82.3 \pm 6.0$ & 0.05 \\
\hline Maximal dimeter, $\mathrm{mm}$ & $27.5 \pm 3.2$ & $30.4 \pm 3.5^{*}$ & $27.4 \pm 2.8$ & 0.001 \\
\hline Mean diameter, $\mathrm{mm}$ & $24.9 \pm 2.6$ & $26.9 \pm 2.5^{*}$ & $25.0 \pm 2.6$ & 0.005 \\
\hline
\end{tabular}

Values are means \pm standard deviations or numbers and percentages in parentheses. ${ }^{*}$ Significant difference between patients with severe aortic stenosis and patients with classic low-flow, lowgradient, severe aortic stenosis (classic LF-LG AS) groups. ${ }^{\dagger}$ Significant difference between severe aortic stenosis and paradoxical LF-LG AS groups. AS, aortic stenosis; AVA, aortic valve area; AVC, aortic valve calcium score; BNP, B-type natriuretic peptide; EDVI, end-diastolic volume index; ESVI, endsystolic volume index; LF-LG, low-flow and low-gradient; InBNP, log-transformed B-type natriuretic peptide; LVEF, left ventricular ejection fraction; LVMI, left ventricular mass index; LVOT, left ventricular outflow tract; MACCE, major adverse cardiac and cerebrovascular event; N/A, not available; PG, pressure gradient; SAC, systemic arterial compliance; VTI, velocity time integral; Zva, valvulo-arterial impedance. 


\begin{tabular}{|c|c|c|c|c|}
\hline Characteristic & $\begin{array}{l}\text { High-gradient } \\
\text { severe AS }\end{array}$ & Classic LF-LG AS & $\begin{array}{l}\text { Paradoxical LF-LG } \\
\text { AS }\end{array}$ & $\begin{array}{l}P \text { - } \\
\text { value }\end{array}$ \\
\hline Perimeter, mm & $79.5 \pm 8.4$ & $85.4 \pm 7.7^{\star}$ & $80.0 \pm 8.3$ & 0.02 \\
\hline Area, $\mathrm{mm}^{2}$ & $481.8 \pm 102.4$ & $554.8 \pm 101.0^{*}$ & $489.5 \pm 99.9$ & 0.01 \\
\hline Sinus of Valsalva, mm & $36.6 \pm 4.5$ & $38.4 \pm 4.7$ & $36.8 \pm 5.0$ & 0.25 \\
\hline ST junction, mm & $30.9 \pm 4.6$ & $31.7 \pm 3.3$ & $31.5 \pm 5.9$ & 0.58 \\
\hline $\begin{array}{l}\text { Ascending aorta tubular } \\
\text { portion, } \mathrm{mm}\end{array}$ & $40.7 \pm 6.3$ & $38.4 \pm 4.6$ & $40.4 \pm 7.7$ & 0.31 \\
\hline \multicolumn{5}{|l|}{ Normalized to BSA } \\
\hline $\mathrm{AVA}_{\mathrm{CT}}, \mathrm{mm}^{2}$ & $51.7 \pm 13.6$ & $60.7 \pm 15.8^{*}$ & $58.0 \pm 15.6^{\dagger}$ & $<0.001$ \\
\hline $\mathrm{AVA}_{\text {plani, }}, \mathrm{mm}^{2}$ & $52.6 \pm 13.5$ & $59.5 \pm 17.5^{\star}$ & $57.8 \pm 15.2^{\dagger}$ & 0.005 \\
\hline \multicolumn{5}{|l|}{ Aortic annulus } \\
\hline Maximal dimeter, mm & $16.8 \pm 2.0$ & $18.2 \pm 2.6^{*}$ & $16.9 \pm 1.7$ & 0.01 \\
\hline Mean diameter, mm & $15.2 \pm 1.6$ & $16.1 \pm 1.9$ & $15.4 \pm 1.6$ & 0.06 \\
\hline Perimeter, mm & $48.7 \pm 5.2$ & $51.1 \pm 5.8$ & $49.2 \pm 5.3$ & 0.12 \\
\hline Area, $\mathrm{mm}^{2}$ & $293.4 \pm 55.5$ & $331.0 \pm 56.6^{*}$ & $299.7 \pm 55.9$ & 0.02 \\
\hline Sinus of Valsalva, mm & $22.4 \pm 2.9$ & $23.0 \pm 2.8$ & $22.6 \pm 2.9$ & 0.65 \\
\hline ST junction diameter, mm & $18.9 \pm 2.9$ & $18.9 \pm 2.1$ & $19.3 \pm 3.2$ & 0.69 \\
\hline $\begin{array}{l}\text { Ascending aorta tubular } \\
\text { portion, } \mathrm{mm}\end{array}$ & $25.0 \pm 4.3$ & $23.0 \pm 2.9$ & $24.8 \pm 4.6$ & 0.15 \\
\hline Surgical valve size, $\mathrm{mm}$ & $22.1 \pm 2.1$ & $23.0 \pm 2.1$ & $22.3 \pm 2.0$ & 0.18 \\
\hline Surgical valve type & & & & N/A \\
\hline CE Magna & 144 & 10 & 18 & \\
\hline ATSAP & 82 & 4 & 8 & \\
\hline \multicolumn{5}{|c|}{ 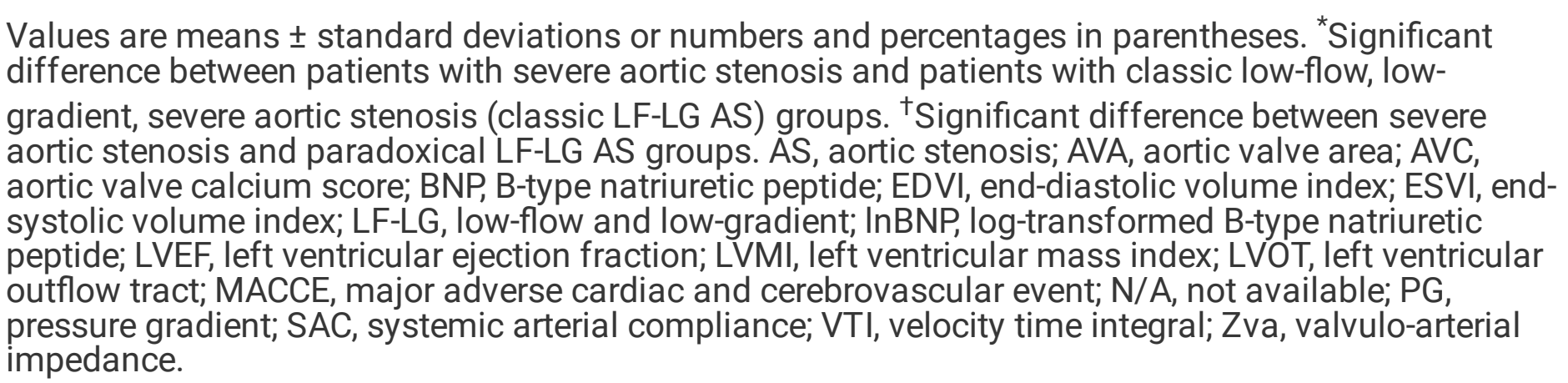 } \\
\hline
\end{tabular}




\begin{tabular}{|c|c|c|c|c|}
\hline Characteristic & $\begin{array}{l}\text { High-gradient } \\
\text { severe AS }\end{array}$ & Classic LF-LG AS & $\begin{array}{l}\text { Paradoxical LF-LG } \\
\text { AS }\end{array}$ & $\begin{array}{l}\mathrm{P} \text { - } \\
\text { value }\end{array}$ \\
\hline Hancock & 82 & 1 & 13 & \\
\hline St. Jude Regent & 80 & 3 & 11 & \\
\hline Others & 50 & 0 & 5 & \\
\hline Operator & & & & 0.47 \\
\hline Operator 1 & 170 & 5 & 18 & \\
\hline Operator 2 & 95 & 2 & 12 & \\
\hline Operator 3 & 81 & 4 & 15 & \\
\hline Operator 4 & 64 & 4 & 7 & \\
\hline Operator 5 & 28 & 3 & 3 & \\
\hline MACCE & $34(7.8)$ & $5(27.8) *$ & $4(7.3)$ & 0.01 \\
\hline Overall mortality & $57(13.0)$ & $6(33.3)$ & $8(14.5)$ & 0.05 \\
\hline Follow-up duration, d & $\begin{array}{l}1517.5(1188.8- \\
2026.5)\end{array}$ & $\begin{array}{l}1134.0(26.0- \\
1682.0)\end{array}$ & $\begin{array}{l}1455.0(1112.0- \\
1944.0)\end{array}$ & 0.007 \\
\hline \multicolumn{5}{|c|}{$\begin{array}{l}\text { Values are means } \pm \text { standard deviations or numbers and percentages in parentheses. *Significant } \\
\text { difference between patients with severe aortic stenosis and patients with classic low-flow, low- } \\
\text { gradient, severe aortic stenosis (classic LF-LG AS) groups. ' }{ }^{+} \text {Significant difference between severe } \\
\text { aortic stenosis and paradoxical LF-LG AS groups. AS, aortic stenosis; AVA, aortic valve area; AVC, } \\
\text { aortic valve calcium score; BNP, B-type natriuretic peptide; EDVI, end-diastolic volume index; ESVI, end- } \\
\text { systolic volume index; LF-LG, Iow-flow and low-gradient; InBNP, log-transformed B-type natriuretic } \\
\text { peptide; LVEF, left ventricular ejection fraction; LVMI, left ventricular mass index; LVOT, left ventricular } \\
\text { outflow tract; MACCE, major adverse cardiac and cerebrovascular event; N/A, not available; PG, } \\
\text { pressure gradient; SAC, systemic arterial compliance; VTI, velocity time integral; Zva, valvulo-arterial } \\
\text { impedance. }\end{array}$} \\
\hline
\end{tabular}

\section{Echocardiography}

LVEF, transaortic peak velocity and PG were lower in the classic LF-LG AS group and reflected the characteristics of LF-LG AS ( $P<0.001$, for all) (Table 1). The ESVI (63.4 vs. $\left.27.7 \mathrm{~mL} / \mathrm{m}^{2}, P<0.001\right)$ and EDVI ( 96.3 vs. $66.8 \mathrm{~mL} / \mathrm{m}^{2}, P<0.001$ ) were significantly larger in classic LF-LG AS, compared to the highgradient severe AS group. SAC was not different among the groups $(P=0.28)$, although Zva was lower in paradoxical LF-LG AS compared to others $(P<0.001)$.

We found that $A V A_{C T} \geq 1.2 \mathrm{~cm}^{2}$ was noted in $27.8 \%$ (5/18) of the patients with classic LF-LG AS (Figure 1). In classic $L F-L G$ AS, $A V A_{\text {echo }}$ was larger in patients with $A V A_{C T} \geq 1.2 \mathrm{~cm}^{2}$ than those with $A V A_{C T}<1.2 \mathrm{~cm}^{2}$ (61.1 vs. $81.9 \mathrm{~mm}^{2}, \mathrm{P}=0.005$ ) (Supplementary Table 2). However, other echocardiography parameters such as LVEF, peak velocity, and PG were not statistically different between subgroups with 
$A_{C A} A_{C T}<1.2 \mathrm{~cm}^{2}$ and $A V A_{C T} \geq 1.2 \mathrm{~cm}^{2}$ (P>0.05, for all). In patients with paradoxical LF-LG AS, AVA echo $_{\text {was }}$ larger in $A V A_{C T} \geq 1.2 \mathrm{~cm}^{2}$ group (68.2 vs. $77.3 \mathrm{~mm}^{2}, P=0.08$ ), but without statistical significance.

\section{Computed tomography}

Interobserver agreements for aortic root measurement on CT are high with the range of ICC from 89.2 97.0. (Supplementary Table 3). The pearson correlation coefficient for $\mathrm{AVA}_{\text {echo }}$ and $\mathrm{AVA}_{\mathrm{CT}}$ was good $(r=0.73, P<0.001) . A V A_{C T}$ is larger than $A V A_{\text {echo }}$ and the mean difference between $A V A_{\text {echo }}$ and $A V A_{C T}$ was $24.1 \mathrm{~mm}^{2}$ ( $95 \% \mathrm{Cl},-8.3$ to $56.4 \mathrm{~mm}^{2}, \mathrm{P}<0.001$ ) (Figure $2 \mathrm{~A}$ and $2 \mathrm{~B}$ ). Comparison of $A \mathrm{AA}_{\text {echo }}$ and $\mathrm{AVA}_{\text {plani }}$ is presented in Supplementary Figure 2. AVC was highest in patients with high-gradient severe $S$, and statistically lower in paradoxical LF-LG AS and moderate AS groups $(P=0.0 .04)$. LVOT dimeter measured on CT was longer in classic LF-LG AS group compared to that in high-gradient severe AS (24.8 vs. 27.1 $\mathrm{mm}, \mathrm{P}=0.003)$. The mean $A V A_{C T}$ was larger in the classic LF-LG AS group, compared to the high-gradient severe AS group (100.8 vs. $84.9 \mathrm{~mm}^{2}, \mathrm{P}=0.001$ ). Normalized aortic annulus maximal diameter was longer (16.8 $\mathrm{mm}$ vs. $18.2 \mathrm{~mm}, \mathrm{P}=0.01)$ and aortic annulus area was larger $\left(293.4 \mathrm{~mm}^{2}\right.$ vs. $\left.331.0 \mathrm{~mm}^{2}, \mathrm{P}=0.02\right)$ in classic LF-LG AS group compared to those in high-gradient severe AS.

In classic LF-LG AS group, mean AVC was higher in $A V A_{C T}<1.2 \mathrm{~cm}^{2}$ group compared to that in $A V A_{C T} \geq 1.2$ $\mathrm{cm}^{2}$ (3912.2 vs. 1360.1, $P=0.002$ ) (Table 2). AVA $A_{\text {plani }}$ was also smaller in $A V A_{C T}<1.2 \mathrm{~cm}^{2}$ group compared to that in $A V A_{C T} \geq 1.2 \mathrm{~cm}^{2}$ (88.2 vs. $\left.129.9 \mathrm{~mm}^{2}, P<0.001\right)$. The normalized annulus sizes and aortic root diameters on CT were not statistically different between $A V A_{C T}<1.2 \mathrm{~cm}^{2}$ and $A V A_{C T} \geq 1.2 \mathrm{~cm}^{2}$ groups $\left(P<0.05\right.$, for all). In paradoxical LF-LG AS patients, LVOT area normalized to BSA (289.3 vs. $345.5 \mathrm{~mm}^{2}$, $\mathrm{P}=0.01)$, normalized sizes of aortic annulus area (292.7 vs. $\left.341.4 \mathrm{~mm}^{2}, \mathrm{P}=0.02\right)$, sinus of Valsalva (22.1 vs. $25.2 \mathrm{~mm}, \mathrm{P}=0.003$ ) and $\mathrm{ST}$ junction (30.6 vs. $36.7 \mathrm{~mm}, \mathrm{P}=0.006$ ) were larger in $A V A_{C T} \geq 1.2 \mathrm{~cm}^{2}$ group, compared to those measured in patients with $\mathrm{AVA}_{\mathrm{CT}}<1.2 \mathrm{~cm}^{2}$. 
Table 2

Subgroups of LF-LG AS according to AVA

\section{Classic LF-LG AS ( $n=18)$}

Characteristic

No. of patients

$(\%)$

Age, years

Male

BSA, $\mathrm{m}^{2}$

Hypertension

Atrial fibrillation

$\mathrm{PCl}$ or $\mathrm{CABG}$

Rheumatic

valvular disease

B-type natriuretic

peptide, $\mathrm{pg} / \mathrm{mL}$

InBNP

Blood urea

nitrogen, $\mathrm{mg} / \mathrm{dL}$

Creatinine, $\mathrm{mg} / \mathrm{dL}$

$2.1 \pm 2.3$

$1118.0(304.8$

$-3066.0)$

$7(53.8)$

$3(23.1)$

$6(46.2)$

$2(15.4)$

$\mathrm{AVA}_{\mathrm{CT}} \geq 1.2$

$\mathrm{cm}^{2}$

$5(27.8)$

$68.6 \pm 7.7$

0.46

$4(80.0)$

1.00

0.12

0.11

$5(100.0)$

1.00

$1(20.0)$

$3(60.0)$

1.00

$0(0.0)$

1.00

738.5

(239.3-

3227.5)

$7.0(5.7-8.0)$

$6.6(5.0-$

7.9)

$25.2 \pm 16.7$

$18.6 \pm 4.7$

$1.9 \pm 1.3$

0.76

$1.0 \pm 0.4$

$4.1(3.5-$

5.2)

$59.0(32.5 \quad 200.5(59.0$

$-305.3)$

$18.8 \pm 8.5 \quad 18.0 \pm 4.7$

0.22

$\mathrm{AVA}_{C T} \geq 1.2$

$\mathrm{cm}^{2}$

47 (85.5)

$67.3 \pm 9.2$

$67.3 \pm 10.9$

0.99

$25(53.2) \quad 6(75.0)$

0.72

$1.6 \pm 0.2$

$1.7 \pm 0.1$

0.34

26 (55.3)

$4(50.0)$

1.00

2 (4.3)

2 (25.0)

0.10

$14(25.5) \quad 0(0.0)$

0.10

$6(12.8)$

$2(25.0)$

0.33

0.38

0.81

$33.8 \pm 8.0$

0.59

$62.8 \pm 5.4$

$61.5 \pm 4.6$

0.53

Peak velocity, $\mathrm{m} / \mathrm{s} \quad 3.6 \pm 0.4$

$3.4 \pm 0.7$

0.37

$3.5 \pm 0.5$

$3.6 \pm 0.6$

0.67

Peak PG, mmHg

$53.5 \pm 11.7$

$48.0 \pm 17.0$

0.44

$49.5 \pm$ 16.0

$53.4 \pm 16.9$

0.53

Mean PG, mmHg $\quad 30.8 \pm 6.8$

$27.2 \pm 10.6$

0.41

$27.7 \pm 9.4$

$29.8 \pm 10.2$

0.59

AS, aortic stenosis; AVA, aortic valve area; $A V A_{C T}$, AVA measured on CT; AVC, aortic valve calcium score; BSA, body surface area; EDVI, end-diastolic volume index; ESVI, end-systolic volume index; LFLG, low-flow and low-gradient; InBNP, log-transformed B-type natriuretic peptide; LVEF, left ventricular ejection fraction; LVMI, left ventricular mass index; LVOT, left ventricular outflow tract; MACCE, major adverse cardiac and cerebrovascular event; VTI, velocity time integral. 


\begin{tabular}{|c|c|c|c|c|c|c|}
\hline \multirow[b]{2}{*}{ LVMI, gm/ m² } & \multicolumn{3}{|c|}{ Classic LF-LG AS $(n=18)$} & \multicolumn{3}{|c|}{ Paradoxical LF-LG AS $(n=55)$} \\
\hline & $151.5 \pm 33.5$ & $\begin{array}{l}144.7 \pm \\
26.3\end{array}$ & 0.69 & $\begin{array}{l}121.5 \pm \\
32.7\end{array}$ & $\begin{array}{l}144.6 \pm \\
50.6\end{array}$ & 0.10 \\
\hline AV TVI, cm & $101.3 \pm 34.2$ & $78.2 \pm 26.9$ & 0.20 & $\begin{array}{l}114.2 \pm \\
28.2\end{array}$ & $98.9 \pm 21.3$ & 0.15 \\
\hline LVOT TVI, cm & $16.0 \pm 4.8$ & $16.2 \pm 3.8$ & 0.92 & $21.4 \pm 3.6$ & $19.7 \pm 3.7$ & 0.24 \\
\hline $\begin{array}{l}\text { LVOT diameter, } \\
\text { mm }\end{array}$ & $22.1 \pm 2.2$ & $21.2 \pm 0.2$ & 0.22 & $21.0 \pm 1.4$ & $22.2 \pm 2.2$ & 0.18 \\
\hline $\begin{array}{l}\text { LVOT } \\
\text { diameter/BSA }\end{array}$ & $12.9 \pm 1.6$ & $13.6 \pm 1.4$ & 0.40 & $13.0 \pm 1.4$ & $13.2 \pm 1.2$ & 0.75 \\
\hline $\mathrm{AVA}_{\mathrm{echo}}, \mathrm{mm}^{2}$ & $61.1 \pm 12.8$ & $81.9 \pm 9.1$ & 0.005 & $\begin{array}{l}68.2 \pm \\
13.6\end{array}$ & $77.3 \pm 12.7$ & 0.08 \\
\hline ESVI & $63.0 \pm 30.0$ & $64.4 \pm 20.0$ & 0.92 & $\begin{array}{l}23.4 \pm \\
10.6\end{array}$ & $30.7 \pm 14.5$ & 0.10 \\
\hline EDVI & $96.4 \pm 32.6$ & $95.9 \pm 21.3$ & 0.98 & $\begin{array}{l}62.0 \pm \\
22.6\end{array}$ & $78.4 \pm 31.5$ & 0.08 \\
\hline $\begin{array}{l}\mathrm{SAC} \\
\mathrm{mL} / \mathrm{m}^{2} / \mathrm{mmHg}\end{array}$ & $0.7 \pm 0.3$ & $0.6 \pm 0.1$ & 0.52 & $0.7 \pm 0.3$ & $1.0 \pm 0.5$ & 0.21 \\
\hline $\begin{array}{l}\mathrm{Zva}, \\
\mathrm{mmHg} / \mathrm{mL} / \mathrm{m}^{2}\end{array}$ & $4.7 \pm 1.7$ & $5.8 \pm 2.2$ & 0.82 & $4.5 \pm 1.4$ & $3.8 \pm 1.5$ & 0.21 \\
\hline \multicolumn{7}{|l|}{ CT findings } \\
\hline Valve morphology & & & 0.28 & & & 0.14 \\
\hline Tricuspid & $9(69.2)$ & $5(100.0)$ & & $26(55.3)$ & $2(25.0)$ & \\
\hline Bicuspid & $4(30.8)$ & $0(0.0)$ & & $21(44.7)$ & $6(75.0)$ & \\
\hline AVC & $\begin{array}{l}3912.2 \\
(2247.0- \\
4496.1)\end{array}$ & $\begin{array}{l}1360.1 \\
(960.1- \\
2108.6)\end{array}$ & 0.002 & $\begin{array}{l}2002.4 \\
(1192.2- \\
2841.6)\end{array}$ & $\begin{array}{l}2584.3 \\
(1306.5- \\
5172.0)\end{array}$ & 0.12 \\
\hline $\mathrm{AVA}_{\mathrm{CT}}$ & $92.4 \pm 15.5$ & $\begin{array}{l}122.7 \pm \\
25.2\end{array}$ & 0.007 & $\begin{array}{l}90.2 \pm \\
22.4\end{array}$ & $\begin{array}{l}117.7 \pm \\
27.9\end{array}$ & 0.003 \\
\hline $\mathrm{AVA}_{\text {plani, }}, \mathrm{mm}^{2}$ & $88.2 \pm 20.0$ & $129.9 \pm 5.4$ & $<0.001$ & $\begin{array}{l}90.1 \pm \\
21.2\end{array}$ & $\begin{array}{l}140.9 \pm \\
13.8\end{array}$ & $<0.001$ \\
\hline
\end{tabular}

$A S$, aortic stenosis; AVA, aortic valve area; $\mathrm{AVA}_{\mathrm{CT}}$, AVA measured on CT; AVC, aortic valve calcium score; BSA, body surface area; EDVI, end-diastolic volume index; ESVI, end-systolic volume index; LFLG, low-flow and low-gradient; InBNP, log-transformed B-type natriuretic peptide; LVEF, left ventricular ejection fraction; LVMI, left ventricular mass index; LVOT, left ventricular outflow tract; MACCE, major adverse cardiac and cerebrovascular event; VTI, velocity time integral. 


\begin{tabular}{|c|c|c|c|c|c|c|}
\hline \multirow[b]{2}{*}{ LVOT area } & \multicolumn{3}{|c|}{ Classic LF-LG AS ( $n=18)$} & \multicolumn{3}{|c|}{ Paradoxical LF-LG AS $(n=55)$} \\
\hline & $587.5 \pm 129.7$ & $\begin{array}{l}572.3 \pm \\
79.9\end{array}$ & 0.81 & $\begin{array}{l}468.9 \pm \\
94.5\end{array}$ & $\begin{array}{l}581.3 \pm \\
94.9\end{array}$ & 0.003 \\
\hline \multicolumn{7}{|l|}{ Aortic annulus } \\
\hline $\begin{array}{l}\text { Maximum } \\
\text { diameter, } \mathrm{mm}\end{array}$ & $30.6 \pm 3.9$ & $30.0 \pm 2.1$ & 0.77 & $27.1 \pm 2.6$ & $29.0 \pm 3.6$ & 0.05 \\
\hline Circularity, \% & $77.6 \pm 7.5$ & $76.9 \pm 4.4$ & 0.84 & $82.0 \pm 5.7$ & $83.8 \pm 7.6$ & 0.18 \\
\hline $\begin{array}{l}\text { Mean diameter, } \\
\mathrm{mm}\end{array}$ & $27.1 \pm 2.9$ & $26.5 \pm 1.4$ & 0.70 & $24.7 \pm 2.3$ & $27.0 \pm 3.0$ & 0.01 \\
\hline Perimeter, mm & $86.0 \pm 8.9$ & $83.8 \pm 3.7$ & 0.46 & $78.7 \pm 7.4$ & $87.3 \pm 10.0$ & 0.01 \\
\hline Area, $\mathrm{mm}^{2}$ & $563.3 \pm 116.1$ & $\begin{array}{l}532.8 \pm \\
45.9\end{array}$ & 0.44 & $\begin{array}{l}475.2 \pm \\
91.3\end{array}$ & $\begin{array}{l}572.9 \pm \\
113.2\end{array}$ & 0.009 \\
\hline $\begin{array}{l}\text { Sinus of Valsalva, } \\
\mathrm{mm}\end{array}$ & $39.3 \pm 4.9$ & $36.1 \pm 3.4$ & 0.20 & $35.9 \pm 4.6$ & $42.0 \pm 4.2$ & 0.001 \\
\hline ST junction, mm & $32.1 \pm 3.5$ & $30.7 \pm 2.8$ & 0.44 & $30.6 \pm 5.1$ & $36.7 \pm 7.7$ & 0.006 \\
\hline $\begin{array}{l}\text { Ascending aorta, } \\
\mathrm{mm}\end{array}$ & $39.5 \pm 4.4$ & $35.5 \pm 4.2$ & 0.10 & $39.6 \pm 7.1$ & $45.1 \pm 9.8$ & 0.06 \\
\hline \multicolumn{7}{|l|}{$\begin{array}{l}\text { Normalized to } \\
\text { BSA }\end{array}$} \\
\hline $\mathrm{AVA}_{\mathrm{CT}}$ & $53.9 \pm 9.4$ & $78.3 \pm 16.1$ & 0.001 & $\begin{array}{l}55.9 \pm \\
14.3\end{array}$ & $70.3 \pm 17.9$ & 0.01 \\
\hline $\mathrm{AVA}_{\text {plani, }}, \mathrm{mm}^{2}$ & $51.5 \pm 12.3$ & $82.7 \pm 6.0$ & $<0.001$ & $\begin{array}{l}55.7 \pm \\
12.7\end{array}$ & $84.1 \pm 10.4$ & $<0.001$ \\
\hline LVOT area, $\mathrm{mm}^{2}$ & $341.2 \pm 69.4$ & $\begin{array}{l}365.5 \pm \\
54.6\end{array}$ & 0.50 & $\begin{array}{l}289.3 \pm \\
55.3\end{array}$ & $\begin{array}{l}345.5 \pm \\
54.4\end{array}$ & 0.01 \\
\hline \multicolumn{7}{|l|}{ Aortic annulus } \\
\hline $\begin{array}{l}\text { Maximal dimeter, } \\
\mathrm{mm}\end{array}$ & $17.9 \pm 2.8$ & $19.2 \pm 2.0$ & 0.35 & $16.8 \pm 1.7$ & $17.4 \pm 1.6$ & 0.37 \\
\hline $\begin{array}{l}\text { Mean diameter, } \\
\mathrm{mm}\end{array}$ & $15.8 \pm 2.0$ & $17.0 \pm 1.7$ & 0.27 & $15.3 \pm 1.6$ & $16.1 \pm 1.6$ & 0.20 \\
\hline Perimeter, mm & $50.2 \pm 6.0$ & $53.6 \pm 5.0$ & 0.28 & $48.7 \pm 4.9$ & $52.0 \pm 6.8$ & 0.10 \\
\hline
\end{tabular}

$A S$, aortic stenosis; AVA, aortic valve area; $\mathrm{AVA}_{\mathrm{CT}}$, AVA measured on CT; AVC, aortic valve calcium score; BSA, body surface area; EDVI, end-diastolic volume index; ESVI, end-systolic volume index; LFLG, low-flow and low-gradient; InBNP, log-transformed B-type natriuretic peptide; LVEF, left ventricular ejection fraction; LVMI, left ventricular mass index; LVOT, left ventricular outflow tract; MACCE, major adverse cardiac and cerebrovascular event; VTI, velocity time integral. 


\begin{tabular}{|c|c|c|c|c|c|c|}
\hline \multirow[b]{2}{*}{ Area, $\mathrm{mm}^{2}$} & \multicolumn{3}{|c|}{ Classic LF-LG AS $(n=18)$} & \multicolumn{3}{|c|}{ Paradoxical LF-LG AS $(n=55)$} \\
\hline & $327.6 \pm 65.3$ & $\begin{array}{l}339.7 \pm \\
26.4\end{array}$ & 0.70 & $\begin{array}{l}292.7 \pm \\
50.4\end{array}$ & $\begin{array}{l}341.4 \pm \\
71.4\end{array}$ & 0.02 \\
\hline Sinus of Valsalva & $22.9 \pm 3.0$ & $23.0 \pm 2.4$ & 0.95 & $22.1 \pm 2.6$ & $25.1 \pm 3.5$ & 0.007 \\
\hline $\begin{array}{l}\text { ST junction } \\
\text { diameter }\end{array}$ & $18.7 \pm 1.9$ & $19.6 \pm 2.5$ & 0.40 & $18.9 \pm 2.5$ & $21.9 \pm 5.3$ & 0.01 \\
\hline $\begin{array}{l}\text { Ascending aorta } \\
\text { tubular portion }\end{array}$ & $23.1 \pm 3.0$ & $22.6 \pm 2.9$ & 0.78 & $24.5 \pm 4.3$ & $26.9 \pm 5.7$ & 0.18 \\
\hline $\begin{array}{l}\text { Surgical valve } \\
\text { size, mm }\end{array}$ & $23.2 \pm 2.3$ & $22.6 \pm 1.7$ & 0.63 & $22.1 \pm 2.1$ & $23.3 \pm 1.7$ & 0.14 \\
\hline $\begin{array}{l}\text { MACCE } \\
\text { (cardiovascular } \\
\text { death) }\end{array}$ & $2(15.4)$ & $3(60.0)$ & 0.10 & $4(8.5)$ & $0(0.0)$ & 1.00 \\
\hline Overall mortality & $4(30.8)$ & $2(40.0)$ & 1.00 & $7(14.9)$ & $1(12.5)$ & 1.00 \\
\hline \multicolumn{7}{|c|}{$\begin{array}{l}\text { AS, aortic stenosis; AVA, aortic valve area; } A V A A_{C T} \text {, AVA measured on CT; AVC, aortic valve calcium } \\
\text { score; BSA, body surface area; EDVI, end-diastolic volume index; ESVI, end-systolic volume index; LF- } \\
\text { LG, Iow-flow and low-gradient; InBNP, log-transformed B-type natriuretic peptide; LVEF, left ventricular } \\
\text { ejection fraction; LVMI, left ventricular mass index; LVOT, left ventricular outflow tract; MACCE, major } \\
\text { adverse cardiac and cerebrovascular event; VTI, velocity time integral. }\end{array}$} \\
\hline
\end{tabular}

\section{Outcome analysis}

MACCE were composed of arrhythmia $(n=6)$, nonfatal cerebrovascular accident (CVA) $(n=10)$, nonfatal myocardial infarction $(n=4)$, heart failure $(n=4)$, reoperation $(n=1)$, and cardiovascular death $(n=18)$. To identify clinical and radiological factors that affect MACCE, cox-proportional hazard regression analysis was performed (Table 3). In univariate analysis, older age, high BNP, high blood urea nitrogen and creatinine, presence of preoperative AF, tricuspid AV, classic LF-LG AS, small AV VTI and LVOT VTI, and small aortic annulus were factors significantly associated with MACCE $(P<0.05$, for all). 
Table 3

Cox proportional hazard regression model for prediction of MACCE

\section{Univariate}

\begin{tabular}{|c|c|c|c|c|}
\hline Parameter & $\mathrm{HR}(95 \% \mathrm{Cl})$ & $\begin{array}{l}\mathrm{P} \text { - } \\
\text { value }\end{array}$ & $\mathrm{HR}(95 \% \mathrm{Cl})$ & P-value \\
\hline Age, years & $1.06(1.02-1.10)$ & 0.005 & $1.04(1.00-1.09)$ & 0.049 \\
\hline Body surface area, $\mathrm{m}^{2}$ & $0.49(0.08-3.15)$ & 0.45 & & \\
\hline $\begin{array}{l}\text { B-type natriuretic peptide, } 10 \\
\mathrm{pg} / \mathrm{mL}\end{array}$ & $1.01(1.003-1.01)$ & $<0.001$ & $\begin{array}{l}1.005(1.003- \\
1.01)\end{array}$ & $<0.001$ \\
\hline $\operatorname{lnBNP}$ & $1.42(1.13-1.77)$ & 0.002 & & \\
\hline Atrial fibrillation, (\%) & $3.22(1.70-6.11)$ & $<0.001$ & $2.75(1.40-5.40)$ & 0.003 \\
\hline LVEF, \% & $0.98(0.96-1.01)$ & 0.20 & & \\
\hline Peak velocity, $\mathrm{m} / \mathrm{s}$ & $0.80(0.57-1.12)$ & 0.19 & & \\
\hline Mean PG, mmHg & $0.99(0.98-1.01)$ & 0.21 & & \\
\hline LVMI, gm/ m² & $0.99(0.99-1.00)$ & 0.18 & & \\
\hline $\mathrm{ESVI}, \mathrm{mL} / \mathrm{m}^{2}$ & $1.01(0.99-1.02)$ & 0.55 & & \\
\hline $\mathrm{EDVl}, \mathrm{mL} / \mathrm{m}^{2}$ & $1.00(0.99-1.01)$ & 0.90 & & \\
\hline $\mathrm{SAC}, \mathrm{mL} / \mathrm{m}^{2} / \mathrm{mmHg}$ & $0.57(0.21-1.53)$ & 0.27 & & \\
\hline $\mathrm{Zva}, \mathrm{mmHg} / \mathrm{mL} / \mathrm{m}^{2}$ & $1.00(0.83-1.20)$ & 0.98 & & \\
\hline Bicuspid aortic valve & $0.40(0.21-0.77)$ & 0.006 & & \\
\hline Classic LF-LG AS & $5.04(1.98-12.84)$ & 0.001 & $5.53(1.74-17.56)$ & 0.004 \\
\hline AVA $_{\text {echo, }}, \mathrm{m}^{2}$ & $1.01(0.99-1.03)$ & 0.60 & & \\
\hline AV VTI, cm & $0.98(0.96-0.99)$ & $<0.001$ & & \\
\hline LVOT VTI, cm & $0.92(0.86-0.99)$ & 0.02 & & \\
\hline $\ln A V C$ & $0.89(0.71-1.09)$ & 0.26 & & \\
\hline
\end{tabular}

\begin{abstract}
AS, aortic stenosis; AVA, aortic valve area; AVC, aortic valve calcium score; EDVI, end-diastolic volume index; ESVI, end-systolic volume index; HR, hazard ratio; LF-LG, low-flow and low-gradient; InBNP, logtransformed B-type natriuretic peptide;LVEF, left ventricular ejection fraction; LVMI, left ventricular mass index; LVOT, left ventricular outflow tract; MACCE, major adverse cardiac and cerebrovascular event; PG, Pressure gradient; SAC, systemic arterial compliance; VTI, velocity time integral; Zva, valvulo-arterial impedance.
\end{abstract}




\begin{tabular}{|c|c|c|c|c|}
\hline & Univariate & & Multivariable & \\
\hline Normalized $\mathrm{AVA}_{\text {plani, }}, \mathrm{mm}^{2}$ & $1.00(0.98-1.03)$ & 0.76 & & \\
\hline Normalized $\mathrm{AVA}_{\mathrm{CT}}, \mathrm{mm}^{2}$ & $1.02(1.00-1.04)$ & 0.50 & & \\
\hline Annulus circularity, \% & $0.34(0.01-18.69)$ & 0.59 & & \\
\hline Aortic annulus area, $\mathrm{cm}^{2}$ & $0.73(0.53-1.01)$ & 0.06 & $0.57(0.40-0.81)$ & 0.002 \\
\hline Surgical valve size, $\mathrm{mm}$ & $0.89(0.77-1.04)$ & 0.13 & & \\
\hline \multicolumn{5}{|l|}{ Surgical valve type } \\
\hline CE Magna & 1 & 0.63 & & \\
\hline ATSAP & $0.55(0.20-1.48)$ & 0.24 & & \\
\hline Hancock & $1.09(0.50-2.38)$ & 0.83 & & \\
\hline St. Jude Regent & $0.63(0.25-1.59)$ & 0.32 & & \\
\hline Others & $0.85(0.31-2.31)$ & 0.75 & & \\
\hline \multicolumn{5}{|l|}{ Operator } \\
\hline Operator 1 & 1 & 0.33 & & \\
\hline Operator 2 & $1.63(0.78-3.38)$ & 0.19 & & \\
\hline Operator 3 & $\begin{array}{l}0.37(0.05-2 . \\
76)\end{array}$ & 0.33 & & \\
\hline Operator 4 & $0.94(0.37-2.41)$ & 0.90 & & \\
\hline Operator 5 & $0.73(0.30-1.78)$ & 0.49 & & \\
\hline \multicolumn{5}{|c|}{$\begin{array}{l}\text { AS, aortic stenosis; AVA, aortic valve area; AVC, aortic valve calcium score; EDVI, end-diastolic volume } \\
\text { index; ESVI, end-systolic volume index; HR, hazard ratio; LF-LG, low-flow and low-gradient; InBNP, log- } \\
\text { transformed B-type natriuretic peptide;LVEF, left ventricular ejection fraction; LVMI, left ventricular } \\
\text { mass index; LVOT, left ventricular outflow tract; MACCE, major adverse cardiac and cerebrovascular } \\
\text { event; PG, Pressure gradient; SAC, systemic arterial compliance; VTI, velocity time integral; Zva, } \\
\text { valvulo-arterial impedance. }\end{array}$} \\
\hline
\end{tabular}

On multivariable analysis, old age (hazard ratio [HR], 1.04, 95\% Cl, 1.00-1.09; $\mathrm{P}=0.049)$, high BNP (HR, 1.005; 95\%Cl, 1.003-1.01 P<0.001), AF (HR, 2.75; 95\% Cl, 1.40-5.40; $\mathrm{P}=0.003)$, classic LF LG AS (HR, $5.53 ; 95 \% \mathrm{Cl}, 1.74-17.56 ; \mathrm{P}=0.004)$, and small aortic annulus area $\left(\mathrm{cm}^{2}\right),[\mathrm{HR}, 0.57 ; 95 \% \mathrm{Cl}, 0.40-0.81$; $\mathrm{P}=0.002]$ ) were factors significantly associated with MACCE (Table 3). Normalized aortic annulus area $\left(\mathrm{cm}^{2}\right)(\mathrm{HR}, 0.40 ; 95 \% \mathrm{Cl}, 0.22-0.74 ; \mathrm{P}=0.004)$ was also a significantly associated factor when the parameter was substitute instead of aortic annulus area in multivariable analysis. When the normalized aortic sinus of Valsalva diameter instead of the aortic annulus size was substituted in the calculation, the 
weight of the other factors remained almost unchanged, while the normalized aortic sinus of Valsalva diameter $(\mathrm{cm})(\mathrm{HR}, 0.30 ; 95 \% \mathrm{Cl}, 0.09-0.98 ; \mathrm{P}=0.04)$ was also identified as a significant factor.

Kaplan-Meier curves indicated significant mortality in the high BNP group (BNP $>700 \mathrm{pg} / \mathrm{mL}$ ) compared to the low BNP group $(P=0.001)$ (Figure $3 A$ ). Furthermore, preoperative $A F$ was also associated with significant mortality (Figure $3 B)(P<0.001)$, and the outcome of classic LF-LG AS was worse in the cumulative survival curve (Figure $3 C)(P=0.001)$.

\section{Discussion}

This study highlights the characteristics of LF-LG AS focusing on CT findings. The AVA $A_{C T}$ and aortic annulus were larger in classic LF-LG AS compared to those in high-gradient severe AS and $27.8 \%$ of classic LF-LG AS patients presented $A V A_{C T} \geq 1.2 \mathrm{~cm}^{2}$. High BNP, preoperative AF, classic LF-LG AS, and smaller aortic root were associated with MACCE after AVR.

Classic LF-LG AS patients demonstrated higher ESVI and EDVI, lower LVEF, larger AVA $A_{\text {echo }}$ and $A V A_{C T}$, and larger aortic annulus compared to high-gradient severe AS. The key messages of this study are demonstrated in Figure 4. In a previous study, patients with severe AS had significantly larger aortic annulus and ST junction diameters compared with those measured in control groups. ${ }^{16}$ This could be attributed to aortic root remodelling: as severe AS progresses ESVI and EDVI increase to compensate, and dilated LV cavity may lead to dilatation of the aortic annulus. Failure to compensate may result in heart failure. Since the BNP was higher in classic LF-LG AS and the group presented poor outcome compared to high-gradient severe AS, classic LF-LG AS may be a compensation failure of high-gradient severe AS. Paradoxical LF-LG AS presented preserved ESVI, EDVI, and LVEF, although $A_{V A}$ echo $_{\text {and }}$ AVA ${ }_{C T}$ were larger than in high-gradient severe AS.

In this study, we used cut-off value of $A V A_{C T}<1.2 \mathrm{~cm}^{2}$ as this value was suggested for severe AS in a previous study. ${ }^{14}$ However, in classic LF-LG AS group, approximately one third of the patients exhibited $A V A_{C T} \geq 1.2 \mathrm{~cm}^{2}$. AVC was lower in the $A V A_{C T} \geq 1.2 \mathrm{~cm}^{2}$ compared to that of $A V A_{C T}<1.2 \mathrm{~cm}^{2}$ group, and in this group, moderate AS patients might be misclassified as severe AS and vice versa. This can also be applied to paradoxical LF-LG patients, despite $14.5 \%$ of these patients presenting $A V A_{C T} \geq 1.2 \mathrm{~cm}^{2}$. Although we could not derive the role of $A V A_{C T}$ in diagnosing LF-LG AS patients, further studies whether $\mathrm{AVA}_{\mathrm{CT}}$ could be used to discriminate true LF-LG AS are would be of value.

The outcome of AS after AVR was associated with preoperative high BNP levels, AF, classic LF-LG AS, and small aortic root. The plasma BNP level was associated with LV dysfunction in AS, and was a well-known predictor of poor outcome in patients with AS overall and after AVR. ${ }^{17-19} \mathrm{AF}$ is also a dominant predictor in both asymptomatic and symptomatic patients with moderate to severe AS, and after AVR. ${ }^{20-22}$ Classic LF-LG AS was associated with worse outcomes after AVR compared those observed in high-gradient AS patients, although LF-LG AS patients have displayed survival benefits with AVR. ${ }^{3}$ Finally, small aortic root 
measured on CT was an independent prognostic factor. This finding should be interpreted cautiously. When AS severity progresses, the increased LV cavity volume may increase the size of the aortic annulus and sinus of Valsalva. However, a small aortic root has also been associated with increased ischemic cardiovascular events and mortality in patients with $\mathrm{AS}^{23}$ possibly reflecting impaired root remodelling process and atherosclerotic changes.

Our study has several limitations. Because this is a retrospective study using a patient cohort that underwent AVR, patients not indicated for surgery due to poor general conditions or comorbidities or who declined operation were not included. The selection bias may affect the outcome assessment, and AVR itself was not used as an outcome parameter. Instead, we used MACCE after AVR. Therefore, the outcomes of this study may not directly infer the outcomes of AS population managed with diverse treatment options. Further studies with AS managed by conservative treatment, surgical AVR, and transcatheter AVR could be of value to evaluate overall outcomes of AS patients. Second, because the small number of LF-LG AS patients, we could not observe the role of $A V A_{C T}$ for reclassification of LF-LG AS. However, we showed the CT characteristics of LF-LG AS: AVA ${ }_{C T}$ and aortic annulus were larger in classic LF-LG AS compared to those in high-gradient severe AS. This finding may be explained by the aortic root remodelling which is associated with the dilated LV. Third, although classic LF-LG patients showed higher overall mortality and a large aortic annulus, a small aortic root was one of the factors associated with MACCE. Both decreased LV function in classic LF-LG AS and impaired aortic root remodelling may contribute to the outcome, respectively, but further studies are necessary to provide more evidences.

In conclusion, classic LF-LG AS presented larger AVA $\mathrm{AT}_{\mathrm{T}}$ and aortic annulus than high-gradient severe AS. Old age, high BNP, AF, classic LF-LG AS and small aortic root on CT were associated with MACCE after AVR. These findings suggest the potential role of cardiac CT in classification and outcome assessment of severe AS.

\section{Methods}

\section{Patients}

This retrospective study was approved by the institutional review board committee of the Asan Medical Center, University of Ulsan College of Medicine (approval number: 2018-0233) and informed consent was waived by the institutional review board committee of the Asan Medical Center, University of Ulsan College of Medicine due to the retrospective nature of observational study. This study was performed in accordance with the Helsinki Declaration. Between June 2011 and Mar 2016, 781 patients underwent surgical AVR. The use of CT was determined mainly by clinician's decision, but in our hospital, cardiac CT examination is generally performed in most of the patients who have performed planned surgical AVR for evaluation of $\mathrm{AV}$ and root morphology based on the guidelines for the appropriate use of cardiac $\mathrm{CT}^{24-27}$. After excluding patients with moderate AS ( $n=24)$, moderate degree of concomitant aortic regurgitation or 
other valvular heart disease $(n=177)$, patients not subjected to preoperative cardiac CT $(n=47)$ or CT without multiphase data $(n=21)$, and a patient with quadricuspid $A V(n=1), 511$ patients were included.

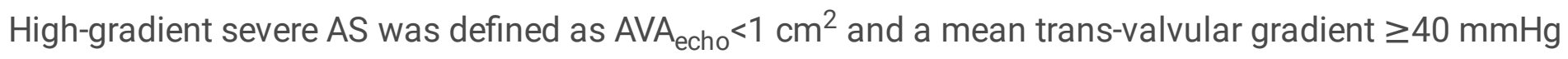
with LVEF $<50 \%$. Classic LF-LG severe AS was defined as $A V A_{\text {echo }}<1 \mathrm{~cm}^{2}$, but with a low-gradient ( $<40$ $\mathrm{mmHg}$ ). Low-gradient severe AS with preserved LVEF was defined as paradoxical LF-LG AS. We classified patients with AS into three groups: 1) high-gradient severe; 2) classic LF-LG; and 3) paradoxical LF-LG AS. Clinical findings including age, body surface area (BSA), hypertension, atrial fibrillation (AF), B-type natriuretic peptide (BNP), echocardiography parameters, and cardiac CT data were collected.

Postoperative echocardiography findings and reported clinical outcomes were comprehensively reviewed. Clinical outcomes included all-cause mortality and MACCE (composite of cardiac death, cerebrovascular accident or stroke, coronary artery revascularization or myocardial infarction, and redo-AVR) were evaluated.

\section{Echocardiography}

Preoperatively, all patients underwent transthoracic echocardiography using commercially available ultrasound machines with 3-5 MHz real-time transducers (iE33, EPIC; Philips Medical Systems, Andover, MA; Vivid 7, E9, General Electric Healthcare, Waukesha, WI, USA). Comprehensive two-dimensional and Doppler images were obtained by expert cardiologists according to American Society of Echocardiography recommendations. ${ }^{28}$ End-systolic volume, end-diastolic volume, and LVEF were obtained with the biplane Simpson method. The maximal aortic jet velocity was recorded with the apical, right parasternal, or suprasternal window that yielded the highest-velocity signal. The maximal and mean PG across the AV were estimated using a modified Bernoulli equation, and the AVA was calculated from the continuity equation. LV mass and LV mass indexed to body surface area calculated by LV cavity dimension and LV wall thickness at end-diastole. Systemic arterial compliance (SAC) was calculated as the ratio of SV index (SVI)/pulse pressure, ${ }^{29}$ and valvulo-arterial impedance (Zva), which is a parameter for global LV load, was defined as (systolic blood pressure + mean net aortic gradient)/SVI. ${ }^{30}$

\section{Cardiac CT protocol and image analysis}

Preoperative cardiac CT was performed using a second-generation dual-source CT scanner (Somatom Definition Flash; Siemens Medical Solutions, Forchheim, Germany). Detailed CT protocol is described in supplementary file 1. Post-processing was conducted using an external workstation (AquariusNet; TeraRecon, Foster City, CA, USA) using multiphase CT data sets reconstructed by a $10 \%$ R-R interval. CT analysis methods are described in supplementary figure 1. CT characteristics such as AV morphology (tricuspid, bicuspid with raphe, and bicuspid without raphe), $\mathrm{AVA}_{\mathrm{CT}}, \mathrm{AVA}$ obtained by planimetry ( $\left.\mathrm{AVA}_{\text {plani }}\right)$, aortic annulus diameter, perimeter, and area, circularity (minimum annulus diameter/maximum annulus diameter $\times 100$ ), and diameters of sinus of Valsalva, sinotubular (ST) junction, and ascending aorta tubular portion were measured by two experienced radiologists in consensus (BLINDED). AVA ${ }_{C T}$ was calculated by using the LV outflow tract (LVOT) area measured on CT in the continuity equation with velocity time integrals (VTI) at LVOT and transaortic flow: $\mathrm{AVA}_{\mathrm{CT}}=\mathrm{LVOT}_{\mathrm{CT}} \times \mathrm{VTI} \mathrm{LVOT}_{\mathrm{LVT}} / \mathrm{VTI}_{\mathrm{AO}}$. 
AVC was defined as a CT density of 130 Hounsfield units or greater confined to AV on non-enhanced cardiac gated images and measured using the methods suggested by Agatston et al. The AVC was measured using a commercially available software (Syngo.via Siemens Healthcare, Berlin, Germany). ${ }^{31}$

Systolic phase with largest AVA (20 30\% RR) was selected and thick multiplanar reconstruction images were used to demarcate the tips of the aortic cusps for measuring $\mathrm{AVA}_{\text {plani. }}$. To evaluate reliability of CT measurements, a third experienced radiologist (BLINDED) measured CT parameters in 100 randomly selected cases and interobserver agreement was determined. Observers were blinded to clinical data including echocardiography findings and operation records.

\section{Statistics}

Continuous variables were expressed as mean \pm standard deviation or median with interquartile range (IQR) and categorical variables are presented as numbers and percentages. Interobserver agreement of CT findings was determined using a two-way random model intra-class correlation coefficient (ICC) with consistency assumption. Comparison of AVA measured on echocardiography ( $A V A_{\text {echo }}$ ) and CT including $\mathrm{AVA}_{\mathrm{CT}}$ and $A V \mathrm{~A}_{\text {plani }}$ was performed using Pearson correlations and Bland-Altman plots were graphed. One way ANOVA with post-hoc (Tukey) test or Kruskall-Wallis test and Chi-square test were used to compare baseline clinical and radiological findings among high-gradient severe AS, classic LF-LG AS, paradoxical LF-LG AS, and moderate AS groups. Bonferroni correction was applied to control the type I error for multiple comparison, and P-value $0.05 / 4=0.0125$ was used for comparing the three groups. Student t-test and Chi-square test were performed to compare two subgroups among the three groups. In LF-LG AS patients, clinical and $C T$ findings for $A V A_{C T}<1.2 \mathrm{~cm}^{2}$ and $A V A_{C T} \geq 1.2 \mathrm{~cm}^{2}$ were compared using the Student t-test and Chi-square test or Fisher's exact test. For the stratification of risk factors for MACCE after AVR, cox proportional hazard models were used. Kaplan-Meier survival curves were drawn for statistically significant factors to predict MACCE. The $95 \%$ confidence intervals (Cls) were calculated and factors with $P<0.10$ were included for multivariable cox regression analysis with enter method. To avoid multicollinearity, one of the aortic root parameters was included in the multivariable analysis among the CT parameters significantly associated with MACCE in univariate analysis. For BNP analysis, a continuous parameter was used and a cut-off of $700 \mathrm{pg} / \mathrm{mL}^{32}$ was set for outcome analysis using Kaplan-Meier curves. ${ }^{19} \mathrm{P}<0.05$ was considered statistically significant, except for multiple dependant variable analyses. Statistical analysis was performed using commercial software (SPSS, version 20; SPSS, Chicago, IL, USA).

\section{Declarations}

\section{Data availability}

All data used during the current study are included in this published article or are available from the corresponding author upon reasonable request.

\section{Contributions}


Conception: H.J.K. and D.K., Data curation: H.J.K., S.L., J.B.K. J-M.S. D-K.K, J-K. S., J-W. K., D.H.Y., Statistical analyses, H.J.K., and H.J.K, Manuscript writing: S.J.C., Y.A., H.J.K., Revising the manuscript: H.J.K. and D.K. All authors approved the final version of this manuscript.

\section{Funding}

This study was supported by a grant (2021IT0006) from the Asan Institute for Life Sciences, Asan Medical Center, Seoul, Korea.

\section{Competing interests}

The authors declare no competing interests.

\section{References}

1. Adda, J. et al. Low-flow, low-gradient severe aortic stenosis despite normal ejection fraction is associated with severe left ventricular dysfunction as assessed by speckle-tracking echocardiography: a multicenter study. Circ Cardiovasc Imaging 5, 27-35, doi:10.1161/CIRCIMAGING.111.967554 (2012).

2. Annabi, M. S. et al. Dobutamine Stress Echocardiography for Management of Low-Flow, LowGradient Aortic Stenosis. Journal of the American College of Cardiology 71, 475-485, doi:10.1016/j.jacc.2017.11.052 (2018).

3. Clavel, M. A., Magne, J. \& Pibarot, P. Low-gradient aortic stenosis. European heart journal 37, 26452657, doi:10.1093/eurheartj/ehw096 (2016).

4. Nishimura, R. A. et al. 2014 AHA/ACC Guideline for the Management of Patients With Valvular Heart Disease: executive summary: a report of the American College of Cardiology/American Heart Association Task Force on Practice Guidelines. Circulation 129, 2440-2492, doi:10.1161/CIR.0000000000000029 (2014).

5. Pibarot, P. \& Dumesnil, J. G. Low-flow, low-gradient aortic stenosis with normal and depressed left ventricular ejection fraction. Journal of the American College of Cardiology 60, 1845-1853, doi:10.1016/j.jacc.2012.06.051 (2012).

6. Dumesnil, J. G. \& Pibarot, P. Low gradient severe aortic stenosis with preserved ejection fraction: don't forget the flow! Rev Esp Cardiol (Engl Ed) 66, 245-247, doi:10.1016/j.rec.2012.10.019 (2013).

7. Minners, J. et al. Inconsistencies of echocardiographic criteria for the grading of aortic valve stenosis. European heart journal 29, 1043-1048, doi:10.1093/eurheartj/ehm543 (2008).

8. Minners, J. et al. Inconsistent grading of aortic valve stenosis by current guidelines: haemodynamic studies in patients with apparently normal left ventricular function. Heart (British Cardiac Society) 96, 1463-1468, doi:10.1136/hrt.2009.181982 (2010).

9. Oh, J. K. et al. Prediction of the severity of aortic stenosis by Doppler aortic valve area determination: prospective Doppler-catheterization correlation in 100 patients. Journal of the American College of 
Cardiology 11, 1227-1234 (1988).

10. deFilippi, C. R. et al. Usefulness of dobutamine echocardiography in distinguishing severe from nonsevere valvular aortic stenosis in patients with depressed left ventricular function and low transvalvular gradients. The American journal of cardiology 75, 191-194 (1995).

11. Koos, R. et al. Aortic valve calcification as a marker for aortic stenosis severity: assessment on 16MDCT. AJR. American journal of roentgenology 183, 1813-1818, doi:10.2214/ajr.183.6.01831813 (2004).

12. Pawade, T. et al. Computed Tomography Aortic Valve Calcium Scoring in Patients With Aortic Stenosis. Circ Cardiovasc Imaging 11, e007146, doi:10.1161/circimaging.117.007146 (2018).

13. Cueff, C. et al. Measurement of aortic valve calcification using multislice computed tomography: correlation with haemodynamic severity of aortic stenosis and clinical implication for patients with low ejection fraction. Heart (British Cardiac Society) 97, 721-726, doi:10.1136/hrt.2010.198853 (2011).

14. Clavel, M. A. et al. Aortic valve area calculation in aortic stenosis by CT and Doppler echocardiography. JACC. Cardiovascular imaging 8, 248-257, doi:10.1016/j.jcmg.2015.01.009 (2015).

15. Halpern, E. J., Mallya, R., Sewell, M., Shulman, M. \& Zwas, D. R. Differences in aortic valve area measured with CT planimetry and echocardiography (continuity equation) are related to divergent estimates of left ventricular outflow tract area. AJR. American journal of roentgenology 192, 16681673, doi:10.2214/ajr.08.1986 (2009).

16. Stolzmann, P. et al. Remodelling of the aortic root in severe tricuspid aortic stenosis: implications for transcatheter aortic valve implantation. European radiology 19, 1316-1323, doi:10.1007/s00330-0091302-0 (2009).

17. Iwahashi, N., Nakatani, S., Umemura, S., Kimura, K. \& Kitakaze, M. Usefulness of plasma B-type natriuretic peptide in the assessment of disease severity and prediction of outcome after aortic valve replacement in patients with severe aortic stenosis. Journal of the American Society of Echocardiography : official publication of the American Society of Echocardiography 24, 984-991, doi:10.1016/j.echo.2011.03.012 (2011).

18. Capoulade, R. et al. Prognostic value of plasma B-type natriuretic peptide levels after exercise in patients with severe asymptomatic aortic stenosis. Heart (British Cardiac Society) 100, 1606-1612, doi:10.1136/heartjnl-2014-305729 (2014).

19. Dahou, A. et al. B-Type Natriuretic Peptide and High-Sensitivity Cardiac Troponin for Risk Stratification in Low-Flow, Low-Gradient Aortic Stenosis: A Substudy of the TOPAS Study. JACC. Cardiovascular imaging 11, 939-947, doi:10.1016/j.jcmg.2017.06.018 (2018).

20. Minamino-Muta, E. et al. Causes of Death in Patients with Severe Aortic Stenosis: An Observational study. Scientific reports 7, 14723, doi:10.1038/s41598-017-15316-6 (2017).

21. Thourani, V. H. et al. Outcomes in 937 Intermediate-Risk Patients Undergoing Surgical Aortic Valve Replacement in PARTNER-2A. The Annals of thoracic surgery 105, 1322-1329, 
doi:10.1016/j.athoracsur.2017.10.062 (2018).

22. Bahler, R. C. et al. Predicting Outcomes in Patients With AsymptomaticModerate to Severe Aortic Stenosis. The American journal of cardiology 122, 851-858, doi:10.1016/j.amjcard.2018.05.027 (2018).

23. Bahlmann, E. et al. Small aortic root in aortic valve stenosis: clinical characteristics and prognostic implications. European heart journal cardiovascular Imaging 18, 404-412, doi:10.1093/ehjci/jew159 (2017).

24. Beck KS, K. J., Choe YH, Hian SK, Hoe J, Hong YJ, et al. . 2017 Multimodality Appropriate Use Criteria for Noninvasive Cardiac Imaging: Expert Consensus of the Asian Society of Cardiovascular Imaging. Cardiovasc Imag Asia 1, 156-165 (2017).

25. Kim, Y. J. et al. Korean guidelines for the appropriate use of cardiac CT. Korean journal of radiology 16, 251-285, doi:10.3348/kjr.2015.16.2.251 (2015).

26. Tsai, I. C. et al. ASCI 2010 appropriateness criteria for cardiac computed tomography: a report of the Asian Society of Cardiovascular Imaging Cardiac Computed Tomography and Cardiac Magnetic Resonance Imaging Guideline Working Group. The international journal of cardiovascular imaging 26 Suppl 1, 1-15, doi:10.1007/s10554-009-9577-4 (2010).

27. Nishimura, R. A. et al. 2017 AHA/ACC Focused Update of the 2014 AHA/ACC Guideline for the Management of Patients With Valvular Heart Disease: A Report of the American College of Cardiology/American Heart Association Task Force on Clinical Practice Guidelines. Journal of the American College of Cardiology, doi:10.1016/j.jacc.2017.03.011 (2017).

28. Mitchell, C. et al. Guidelines for Performing a Comprehensive Transthoracic Echocardiographic Examination in Adults: Recommendations from the American Society of Echocardiography. Journal of the American Society of Echocardiography : official publication of the American Society of Echocardiography 32, 1-64, doi:10.1016/j.echo.2018.06.004 (2019).

29. de Simone, G. et al. Stroke volume/pulse pressure ratio and cardiovascular risk in arterial hypertension. Hypertension (Dallas, Tex. : 1979) 33, 800-805 (1999).

30. Briand, M. et al. Reduced systemic arterial compliance impacts significantly on left ventricular afterload and function in aortic stenosis: implications for diagnosis and treatment. Journal of the American College of Cardiology 46, 291-298, doi:10.1016/j.jacc.2004.10.081 (2005).

31. Agatston, A. S. et al. Quantification of coronary artery calcium using ultrafast computed tomography. Journal of the American College of Cardiology 15, 827-832, doi:10.1016/0735-1097(90)90282-t (1990).

32. Sakurai, S. et al. Brain natriuretic peptide facilitates severity classification of stable chronic heart failure with left ventricular dysfunction. Heart (British Cardiac Society) 89, 661-662, doi:10.1136/heart.89.6.661 (2003).

\section{Figures}




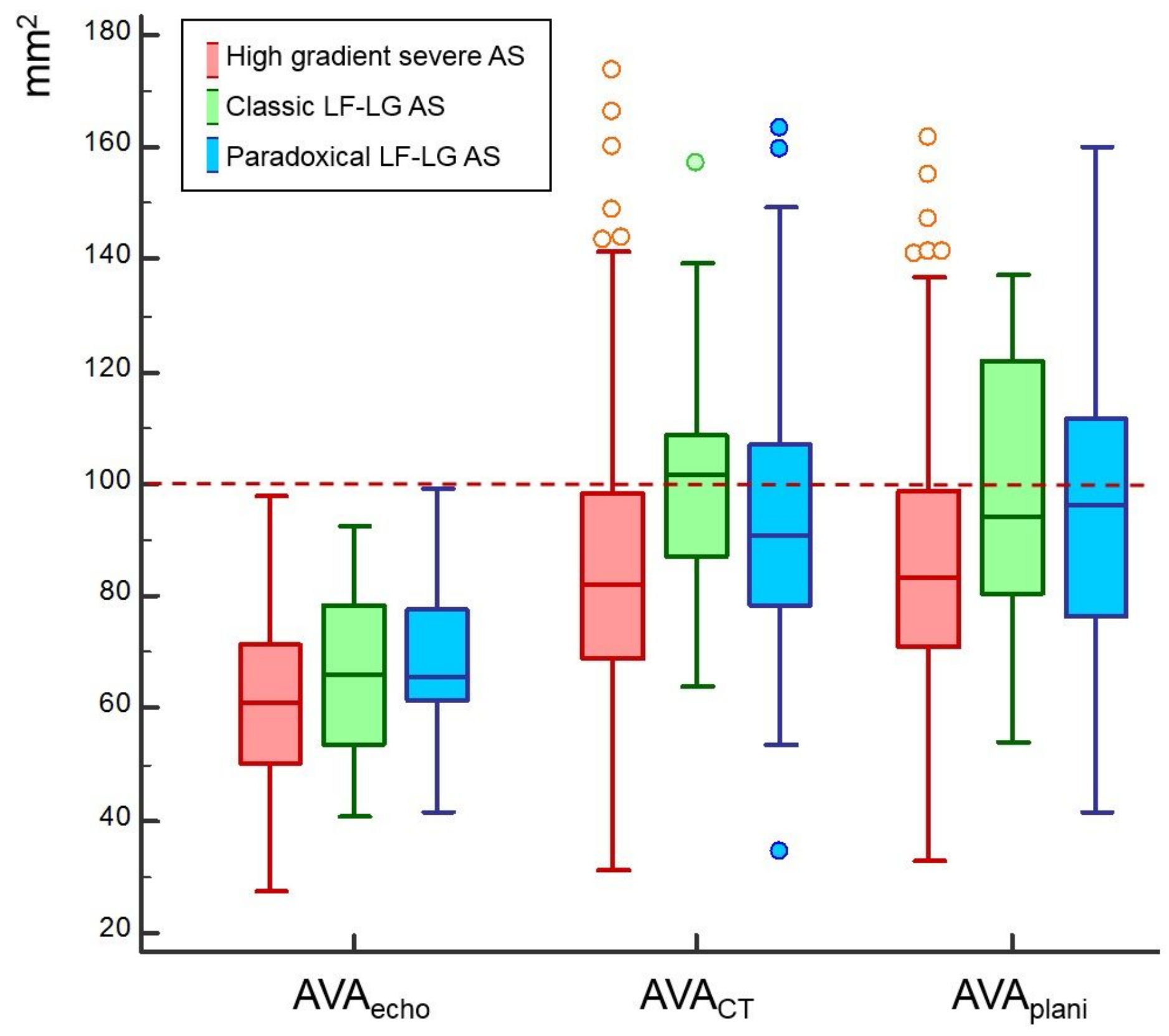

Figure 1

Box plot to demonstrate the distribution of $\mathrm{AVA}_{\mathrm{echo}}, \mathrm{AVA}_{\mathrm{CT}}$, and $\mathrm{AVA}_{\mathrm{plani}}$ according to categories of aortic stenosis

AS, aortic stenosis; AVA, aortic valve area; LF-LG, low-flow and low-gradient 


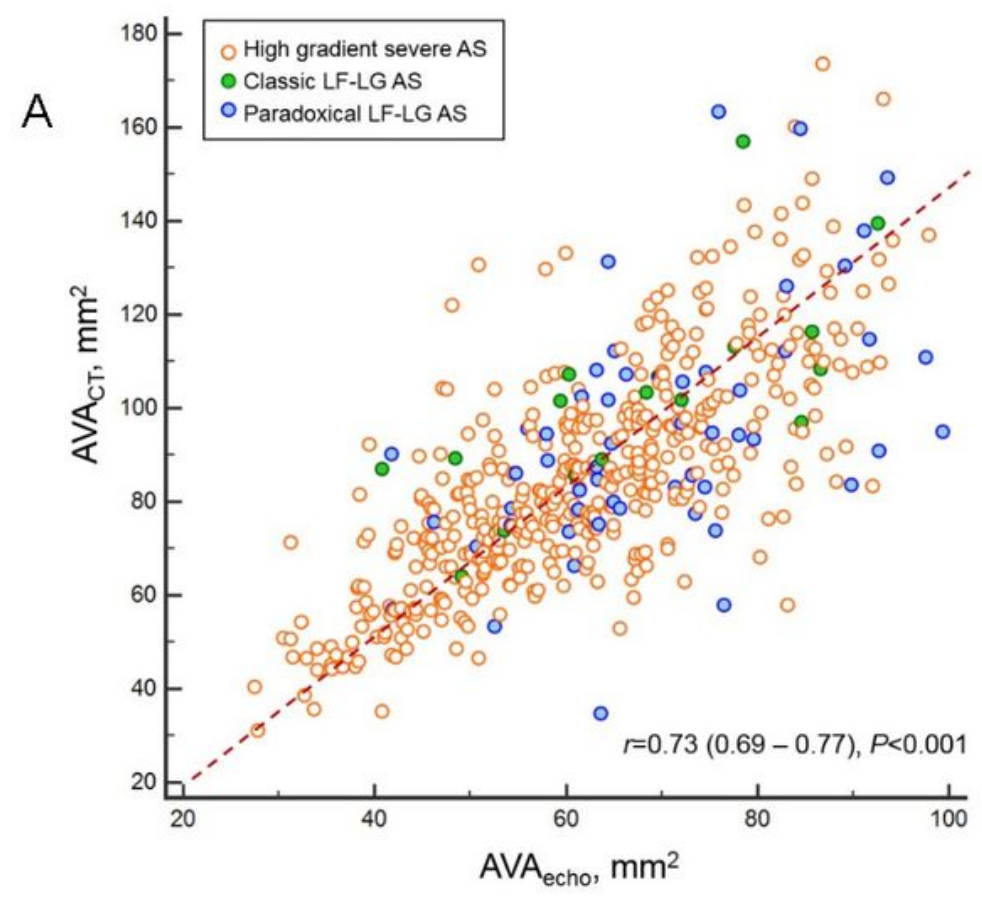

B

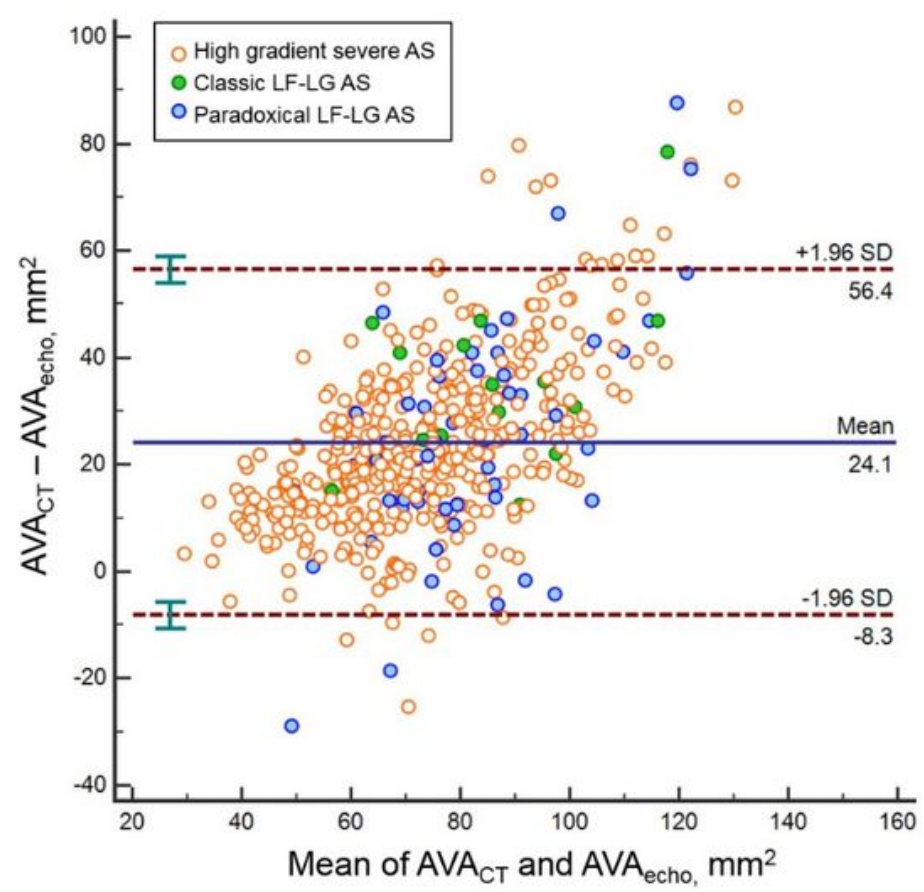

Figure 2

(a) Pearson correlation analysis result and (b) Bland-Altman plot to comparison of AVA $A_{C T}$ and $A V A_{\text {echo }}$ AS, aortic stenosis; AVA, aortic valve area; LF-LG, low-flow and low-gradient 

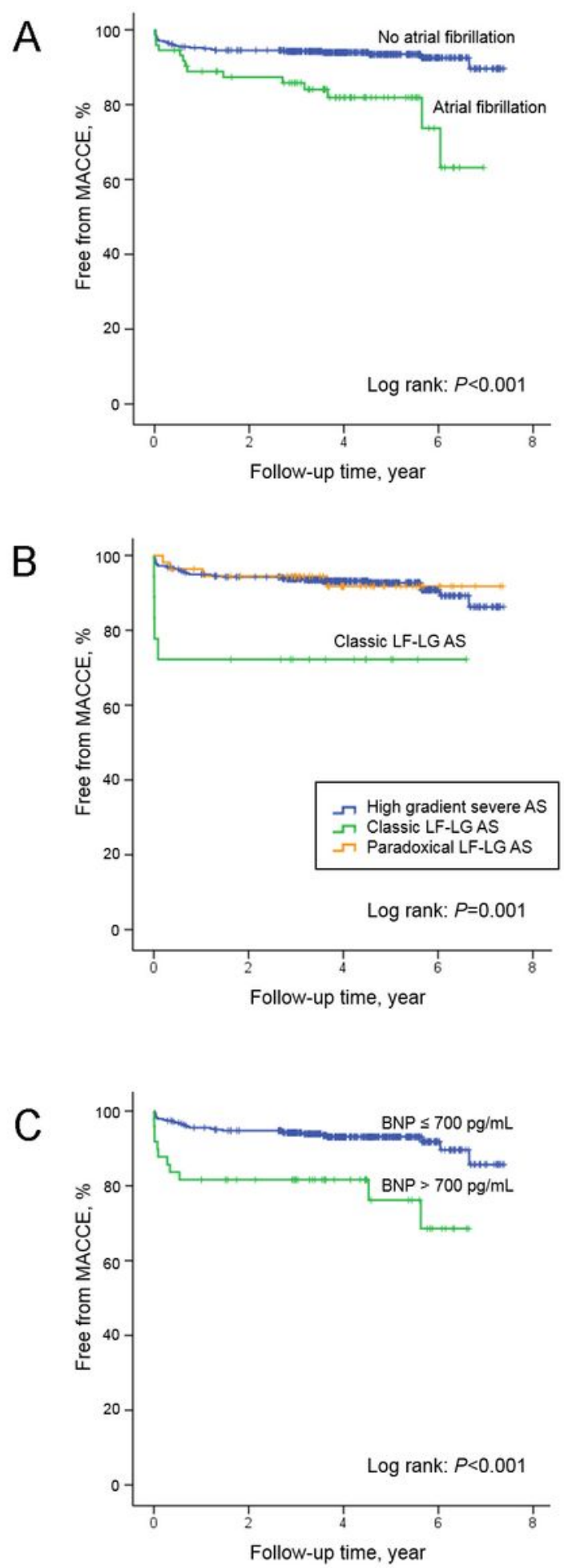

\section{Figure 3}

Survival according to (a) B-type natriuretic peptide, (b) presence of atrial fibrillation, and (c) categories of aortic stenosis

AS, aortic stenosis; LF-LG, low-flow and low-gradient 


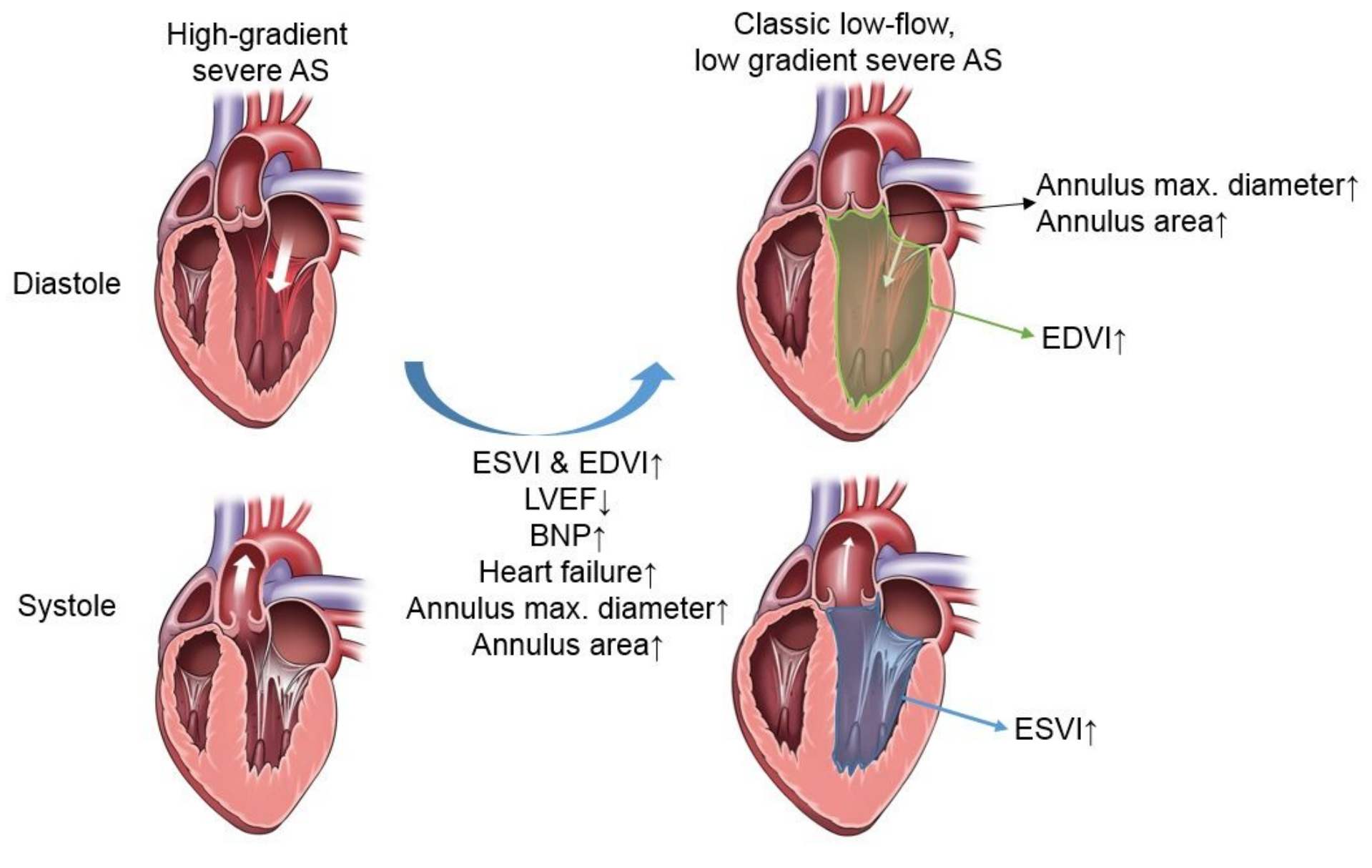

Figure 4

Characteristics of classic LF-LG AS

AS, aortic stenosis; BNP, B-type natriuretic peptide; EDVI, end-diastolic volume index; ESVI, end-systolic volume index; LVEF, left ventricular ejection fraction;

\section{Supplementary Files}

This is a list of supplementary files associated with this preprint. Click to download.

- LFLGSupplementaryFile.docx 